\title{
Radiation Exposure in the Lower Atmosphere during Different Periods of Solar Activity
}

\author{
Anastasia Tezari ${ }^{1,2}$, Pavlos Paschalis ${ }^{1}$, Argyris Stassinakis ${ }^{1}$, Helen Mavromichalaki ${ }^{1, *}$, Pantelis Karaiskos ${ }^{3}$, \\ Maria Gerontidou ${ }^{1}{ }^{\circledR}$, Dimitris Alexandridis ${ }^{1}$, Anastasios Kanellakopoulos ${ }^{4,5}$, Norma Crosby ${ }^{6}$ and \\ Mark Dierckxsens ${ }^{6}$
}

1 Athens Cosmic Ray Group, Faculty of Physics, National and Kapodistrian University of Athens, 15784 Athens, Greece; anatez@med.uoa.gr (A.T.); ppaschalis@phys.uoa.gr (P.P.); a-stasinakis@phys.uoa.gr (A.S.); mgeront@phys.uoa.gr (M.G.); dalexand@med.uoa.gr (D.A.)

2 Eugenides Foundation, 17564 Athens, Greece

3 Medical Physics Laboratory, Faculty of Medicine, National and Kapodistrian University of Athens, 11517 Athens, Greece; pkaraisk@med.uoa.gr

4 Instituut voor Kern-en Stalingsfysica, KU Leuven, 3001 Leuven, Belgium; anastasios.kanellakopoulos@hesge.ch

5 HEPIA/HES-SO, University of Applied Sciences of Western Switzerland, 1202 Geneva, Switzerland

6 Royal Belgian Institute for Space Aeronomy, 1180 Brussels, Belgium; Norma.Crosby@aeronomie.be (N.C.); mark.dierckxsens@aeronomie.be (M.D.)

* Correspondence: emavromi@phys.uoa.gr; Tel.: +30-(210)-727-6890

Citation: Tezari, A.; Paschalis, P.; Stassinakis, A.; Mavromichalaki, H.; Karaiskos, P.; Gerontidou, M.; Alexandridis, D.; Kanellakopoulos, A.; Crosby, N.; Dierckxsens, M. Radiation Exposure in the Lower Atmosphere during Different Periods of Solar Activity. Atmosphere 2022, 13, 166. https://doi.org/10.3390/ atmos13020166

Academic Editors: Tatiana

A. Egorova and Sergey Pulinets

Received: 29 November 2021

Accepted: 18 January 2022

Published: 20 January 2022

Publisher's Note: MDPI stays neutral with regard to jurisdictional claims in published maps and institutional affiliations.

Copyright: () 2022 by the authors. Licensee MDPI, Basel, Switzerland. This article is an open access article distributed under the terms and conditions of the Creative Commons Attribution (CC BY) license (https:// creativecommons.org/licenses/by/ $4.0 /)$.

\begin{abstract}
In recent years, there has been a huge increase in air travel, both for business and leisure. For this reason, entities such as the European Commission and the International Committee on Radiological Protection have provided several recommendations for the radiation protection of aviation crews and frequent flyers, as well as highlighted the need for accurate tools for radiation assessment in the atmosphere. With a focus on the most frequent commercial flying altitudes, this work has performed dosimetry calculations in the lower atmosphere of Earth for different values of cut-off rigidity, covering the recent solar cycles 23 and 24. Results are based on Monte Carlo simulations performed with the validated Geant 4 software application Dynamic Atmospheric Shower Tracking Interactive Model Application (DYASTIMA) and its extension, DYASTIMA-R.
\end{abstract}

Keywords: ambient dose equivalent; cosmic radiation; aviation; solar cycle

\section{Introduction}

Cosmic radiation is constantly bombarding the Earth's atmosphere, making it an important contributor to the natural background radiation on the Earth's surface. However, the exposure in the higher atmospheric layers and in space may vary significantly $[1,2]$. Therefore, a different approach is needed as far as the radiation assessment of aircrews is concerned, especially since the exposure levels of commercial aircraft crews are higher than any other occupationally exposed working group [3]. For this reason, the European Commission, taking into consideration the recommendations made by the International Committee on Radiological Protection (ICRP) and the International Commission on Radiation Units and Measurements (ICRU), have adopted a legislative framework for the radiation protection of aviators as far as their exposure to cosmic radiation is concerned [4]. According to the ALARA principle ("as low as reasonably achievable"), the radiation exposure of aviators should be minimized according to the existing socioeconomic conditions, which can be quite restrictive as far as the aviation industry is concerned. The principles of radiation protection are of great importance, especially during extreme events. Thus, there is a need for reliable, user-friendly, and easily accessible models and tools for the performance of dosimetry calculations in the atmosphere [5]. The scientific community 
has developed several suitable tools so far, such as AVIDOS [6], SIEVERT [7], CARI [8], NAIRAS [9] and SPENVIS [10].

In this context, the Athens Cosmic Ray Group has built a new software application called Dynamic Atmospheric Shower Tracking Interactive Model Application (DYASTIMA) [11], based on Geant4 [12-14]. DYASTIMA is a software application for the simulation of air showers and cosmic ray secondary particles cascades inside the atmosphere of Earth. Its new feature, DYASTIMA-R $[15,16]$, allows for the calculation of radiation doses (dose rate and equivalent dose rate) as a function of different parameters (atmospheric altitudes, geographic coordinates, and phases of solar activity). DYASTIMA has been validated according to international standards, as proposed in the documents by ICRP [17] and ICRU [18], and therefore can provide accurate dosimetry calculations for the exposure of aircrews due to cosmic radiation [19-21]. Results of specific DYASTIMA/DYASTIMA-R simulation scenarios are provided by the Space Radiation Expert Service Centre (R-ESC) on the European Space Agency Space Weather Service (ESA SWE) Portal as a federated product (https://swe.ssa.esa.int/dyastima-federated) (accessed on 17 January 2022), while the software application and the Software User's Manual [22] are provided through the Athens Neutron Monitor Station (A.Ne.Mo.S.) Portal (http:/ / cosray.phys.uoa.gr/index.php/dyastima) (accessed on 17 January 2022).

In this work, radiation dosimetry calculations are performed with DYASTIMA-R for a time period of twenty-five years, covering the two recent solar cycles, 23 (1996-2008) and 24 (2009-2019), estimating the exposure of aviation crews and frequent flyers to cosmic radiation. The years 2001 and 2014 are the solar maximum of solar cycle 23 and 24, respectively, while the period 2007 to 2009 corresponds to an unusual period of minimum solar activity [23]. These calculations concern the lower atmosphere of Earth, as well as the most usual flying levels (FL) of commercial aircrafts. The aforementioned Monte Carlo simulations have been performed for different geographic locations, covering the whole range of magnetic cut-off rigidity thresholds $(\mathrm{Rc}=0-17 \mathrm{GV})$. The simulation parameters and results are analyzed and thoroughly discussed, while future steps are also presented.

\section{Technical Analysis}

To perform a simulation with DYASTIMA/DYASTIMA-R, several input parameters should be defined via a user-friendly graphical interface to fully describe the simulation environment as well as all the physical interactions taking place [11]. These parameters concern the planet's characteristics, the volume of the simulation, the appropriate physics list, the type and material of the human phantom (for example cylindrical phantom or ICRU sphere), and the number of events and iterations $[15,16,22]$. The definition of both the atmospheric composition and the temperature as a function of the atmospheric altitude, i.e., the atmospheric profile, are cornerstones of the simulation. In this work, the International Standard Atmosphere (ISA) model has been used to simulate the Earth's atmosphere [24], as it best describes the average atmospheric conditions in middle latitudes. This is a well-known model and widely accepted by the scientific community for aviation studies. Nevertheless, DYASTIMA provides the ability to replace it with other models used for the description of the atmosphere.

Another very important aspect of a DYASTIMA simulation is the definition of the spectrum of the primary cosmic radiation particles reaching the top of the Earth's atmosphere. In this context, this corresponds to an atmospheric altitude of $85 \mathrm{~km}$, as the Earth's troposphere, stratosphere, and thermosphere contain approximately $99 \%$ of the total atmospheric mass, while $99.99997 \%$ of the mass of the atmosphere is below $100 \mathrm{~km}$ (Kármán Line) [25]. The differential flux of primary cosmic radiation as a function of the energy of primary cosmic particles for the scenarios presented in this work is according to the ISO15390 model [26]. ISO15390 is based on semi-empirical galactic cosmic radiation models, enhanced by yearly average values of the Wolf number, in order to take into account the 11-year solar cycle variability. 
To achieve higher accuracy, the primary spectra of the elements $\mathrm{H}, \mathrm{He}, \mathrm{C}, \mathrm{O}, \mathrm{Si}$ and $\mathrm{Fe}$ were applied, since they are observed in the abundances of cosmic radiation [27]. In order to take into account the effect of the geomagnetic field, the values of the vertical cut-off rigidity threshold Rc for different geographical coordinates based on the International Geomagnetic Reference Field (IGRF) were used [28,29]. The corresponding components of the magnetic field were obtained by the National Oceanic and Atmospheric Administration (https:/ / www.ngdc.noaa.gov/geomag/) (accessed on 28 December 2021).

An example of the primary cosmic ray spectrum for the polar region $(\mathrm{Rc}=0 \mathrm{GV})$ for solar minimum 2009 (upper panel) and solar maximum 2014 (lower panel), as used in the DYASTIMA/DYASTIMA-R simulations, is illustrated in Figure 1. During solar maximum conditions (2014), a shift of the maximum differential flux of the primary particles towards higher energies is observed, due to the occurrence of intense solar phenomena, such as solar flares and coronal mass ejections, resulting in particles of higher energy. In contrast, the differential flux reaches higher maximum values during solar minimum (2009), which is due to the anti-correlation of solar activity with the intensity of the cosmic rays $[1,30]$.
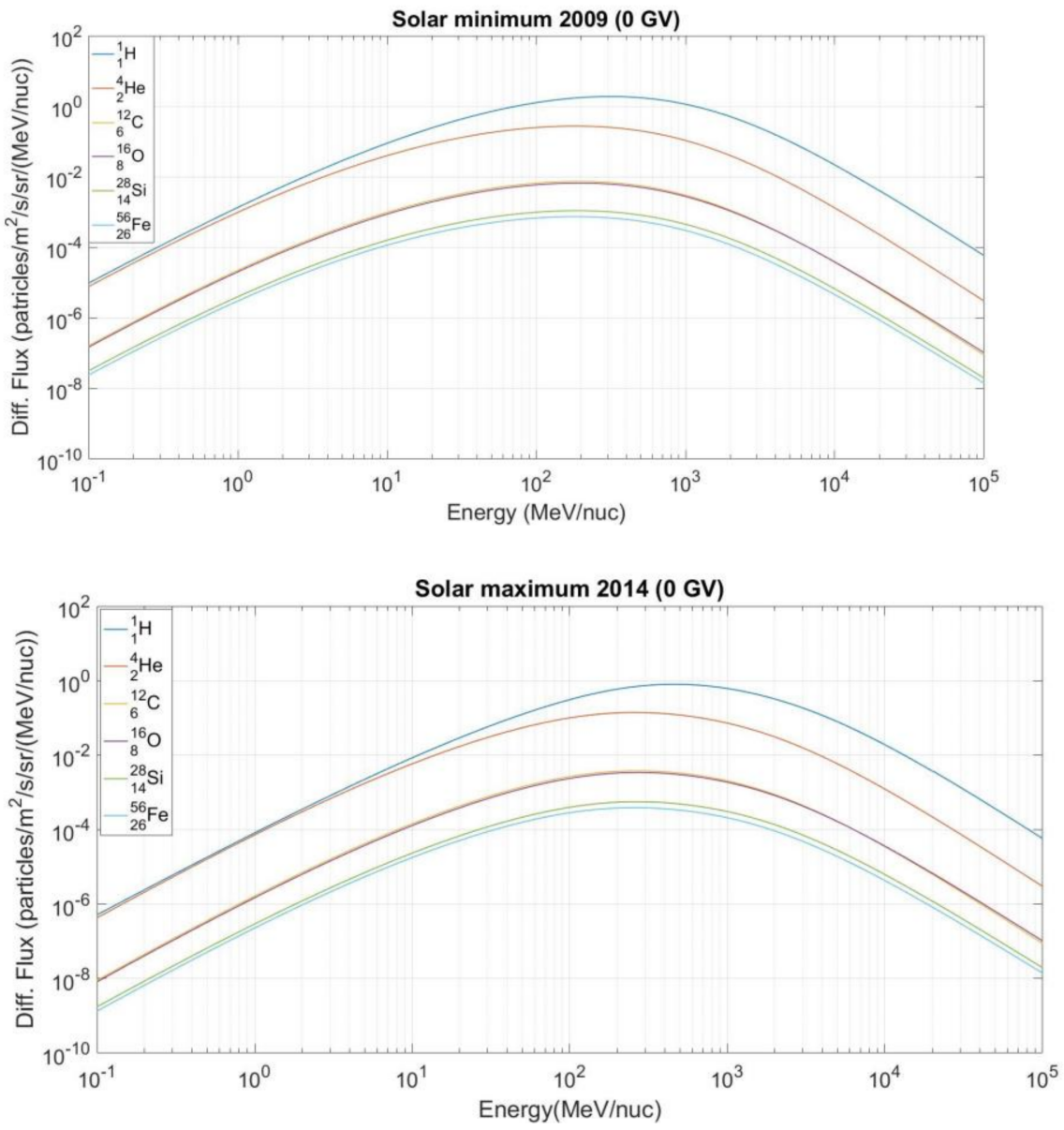

Figure 1. Differential flux as a function of energy for various elements for the years 2009 (solar minimum) and 2014 (solar maximum).

Similar behavior can also be identified in the deposit of ionizing energy due to secondary cosmic radiation cascades in the atmosphere for different solar activity phases. The energy deposit for the years 2009 and 2014 for various cut-off rigidity thresholds, as calculated by DYASTIMA simulations, is presented in Figure 2. The energy deposit to the atmospheric showers begins at an altitude of $45 \mathrm{~km}$, maximizes at $12 \mathrm{~km}$, and then reduces exponentially towards the Earth's surface. As expected, the maximum deposition of energy occurs at polar geographic latitudes, where the cut-off rigidity threshold obtains its minimum value $(\mathrm{Rc}=0 \mathrm{GV})$. Respectively, the energy deposit decreases towards the middle 
latitude and equatorial regions $(\mathrm{Rc}=15 \mathrm{GV})$, as the cut-off rigidity threshold increases. This is due to the geomagnetic field properties, as low-energy particles are deflected in the equator, while they can be recorded in the poles [31]. This phenomenon is also observed in both periods of minimum and maximum solar activity. It is also noted that during 2009, the energy deposit is greater compared to 2014, due to the well-known negative correlation of solar activity to cosmic ray intensity.
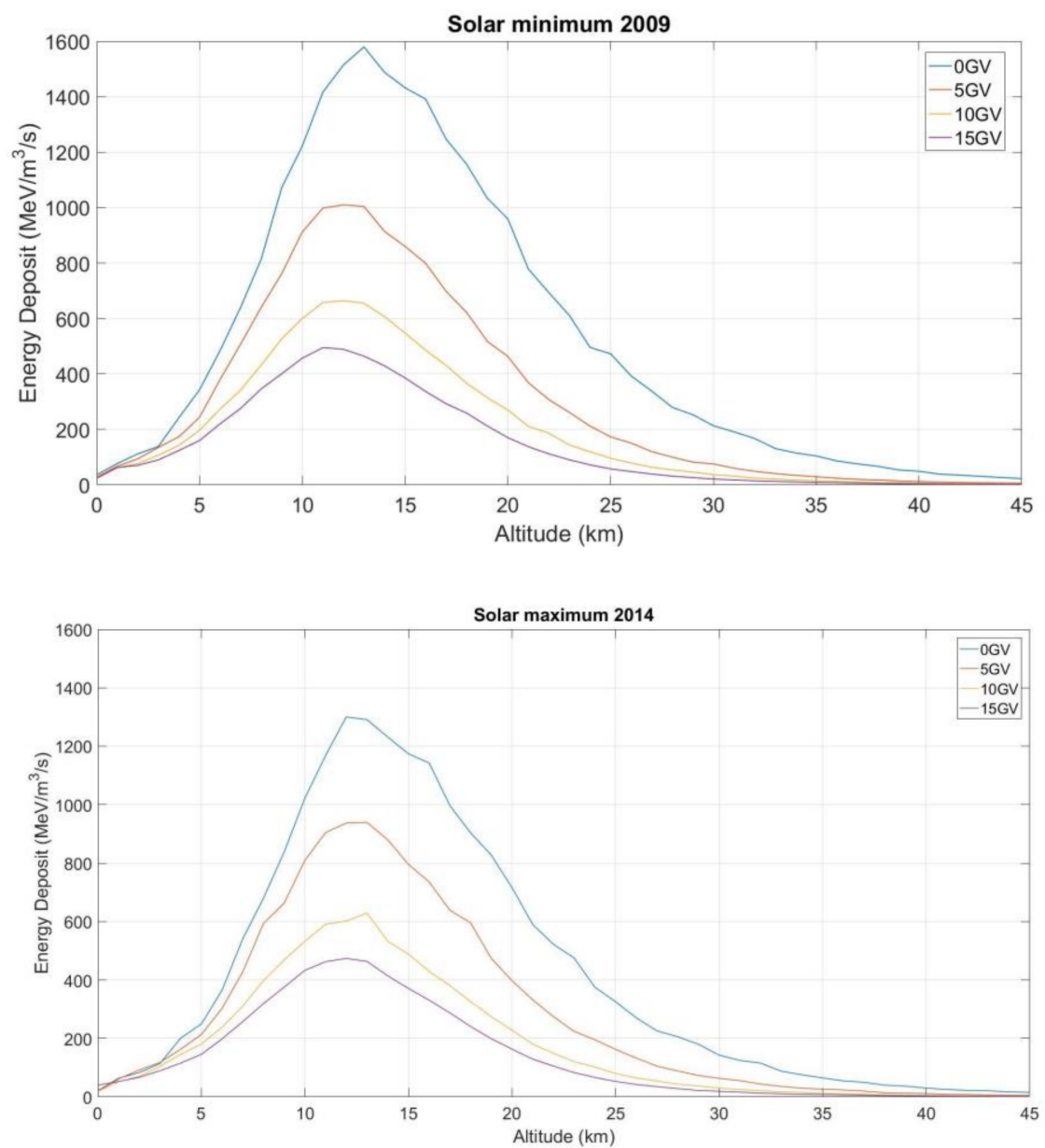

Figure 2. Energy deposit as a function of atmospheric altitude for the years 2009 (solar minimum) and 2014 (solar maximum).

Based on these results, simulations were performed with the extension DYASTIMA-R in order to estimate the ambient dose equivalent rate $\mathrm{dH}^{*}(10) / \mathrm{dt}$ that may be acquired by aircrews as well as frequent flyers in different atmospheric altitudes. The quantity $\mathrm{dH}^{*}$ $(10) / \mathrm{dt}$ as a function of the altitude in the lower atmosphere $(0-20 \mathrm{~km})$ for different values of cut-off rigidity ( $0 \mathrm{GV}, 5 \mathrm{GV}, 10 \mathrm{GV}, 15 \mathrm{GV})$ for solar minimum (2009) and solar maximum conditions is presented in Figure 3. The three vertical lines highlight the frequent flying altitudes of commercial aircrafts (FL310 $=9.45 \mathrm{~km} / 31,000 \mathrm{ft}$, FL350 $=10.67 \mathrm{~km} / 350,000 \mathrm{ft}$, FL390 $=11.89 \mathrm{~km} / 39,000 \mathrm{ft}$ ).

It should be pointed out that the exposure is practically negligible and independent of the cut-off rigidity threshold for the lower altitudes $(0-4 \mathrm{~km})$. As expected, the dose rate increases with the atmospheric altitude, especially for a rigidity range of $0 \mathrm{GV}$ to $5 \mathrm{GV}$. It is also noted that there is a linear increment of the dose rate between FL310 and FL390. In addition, the value of $\mathrm{dH}^{*}(10) / \mathrm{dt}$ doubles in the range of FL310 to FL390. 

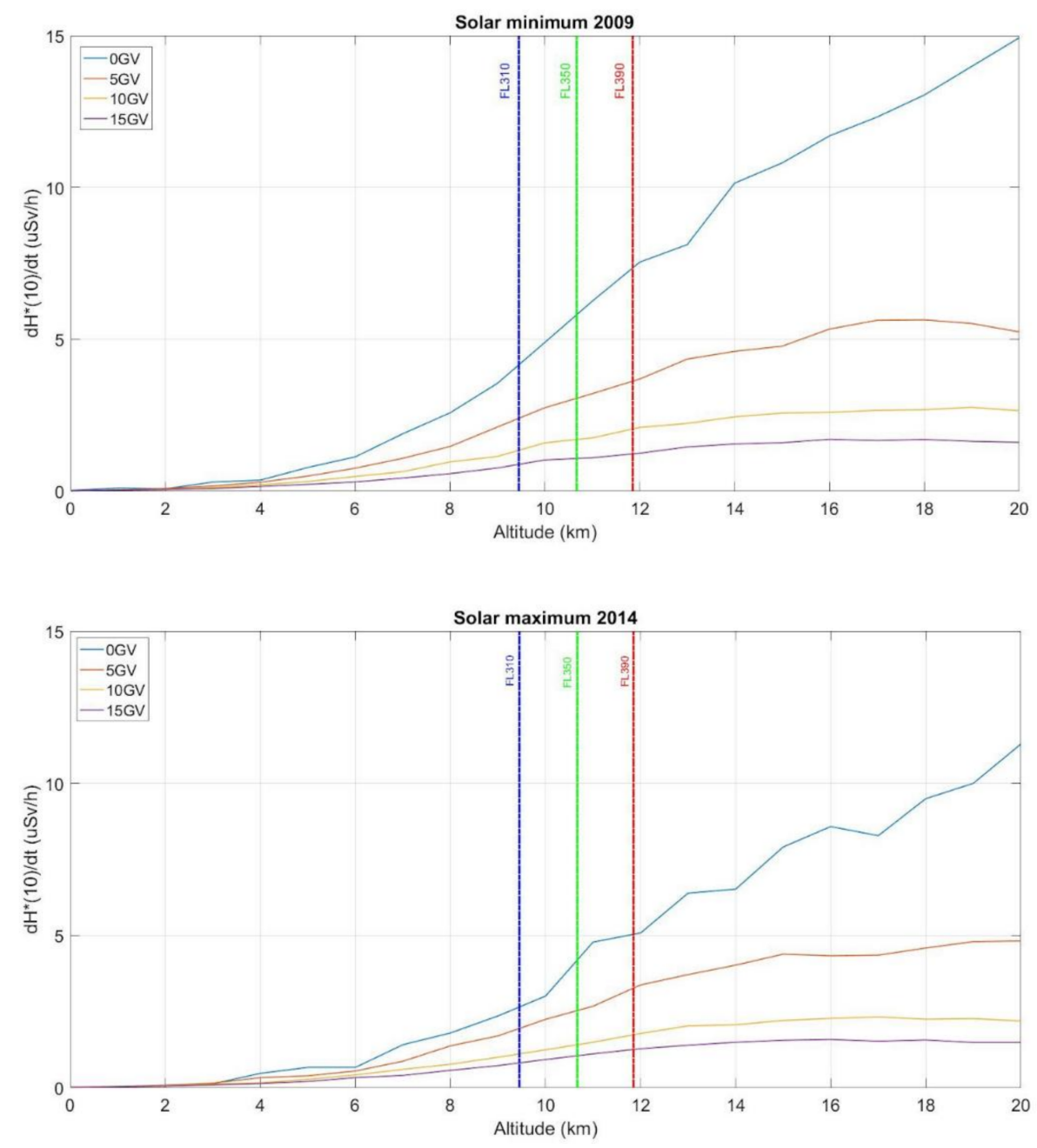

Figure 3. Ambient dose equivalent rate vs. altitude in the lower atmosphere for the years 2009 (solar minimum) and 2014 (solar maximum).

The ambient dose equivalent rate has been calculated as a function of the cut-off rigidity threshold for 2009 and 2014 and is presented in Figure 4. Furthermore, the simulation results are fitted with an exponential curve. According to the results of Figure 4, the fitted curves have high accuracy as the parameter of $R^{2}$ is above 0.97 in all cases.

Considering that each geographic location corresponds to a specific cut-off rigidity threshold [28,29], the dose rate as a function of geographic longitude and latitude is presented in Figure 5, for three flying altitudes (FL310, FL350, FL390). As expected, dH * (10)/dt increases exponentially from the equator to the poles.

Finally, radiation dosimetry calculations have been performed with DYASTIMA-R for the two recent solar cycles, covering a time period of twenty-five years (1996-2019). The dose rate dependence on the 11-year solar cycle variation for various cut-off rigidity thresholds $(0 \mathrm{GV}, 5 \mathrm{GV}, 10 \mathrm{GV}, 15 \mathrm{GV})$ and for three flying altitudes (FL310, FL350, FL390) is displayed in Figure 6, where the simulation results are fitted with 4th order polynomial curves. 
Due to the well-known anti-correlation observed between cosmic radiation intensity and solar activity, it is expected that the dose rate will exhibit a similar behavior, since it is utterly dependent on the intensity of the incoming cosmic ray particles [31]. Therefore, during the extended solar minimum (2006-2009), the maximum dose rate is observed [32,33]. According to Figure 6, the ambient dose equivalent rate is affected by the solar cycle only for low rigidity values, i.e., Rc $<5 \mathrm{GV}$. It is therefore observed that dose rate is independent of the solar cycle phases in the equatorial regions.

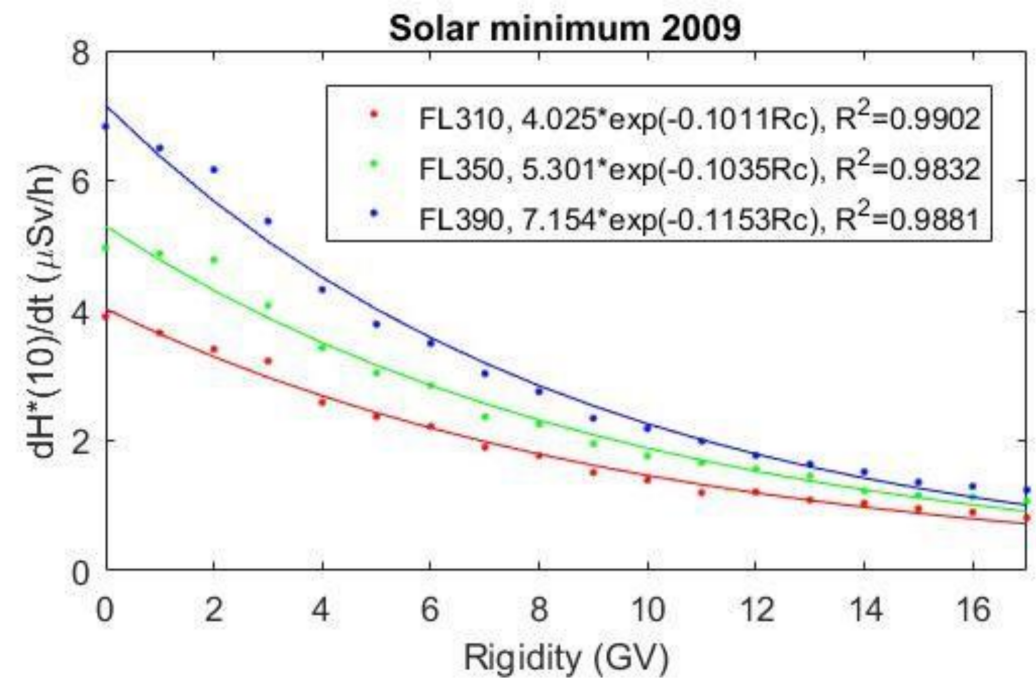

Solar maximum 2014

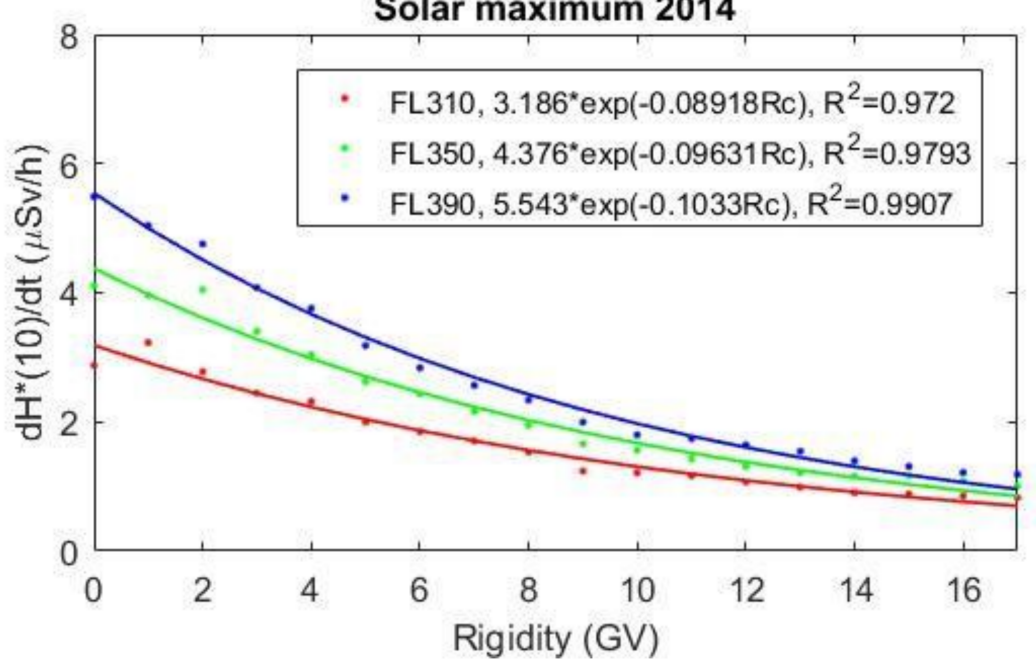

Figure 4. Ambient dose equivalent rate vs. of the cut-off rigidity threshold for the years 2009 (solar minimum) and 2014 (solar maximum). 

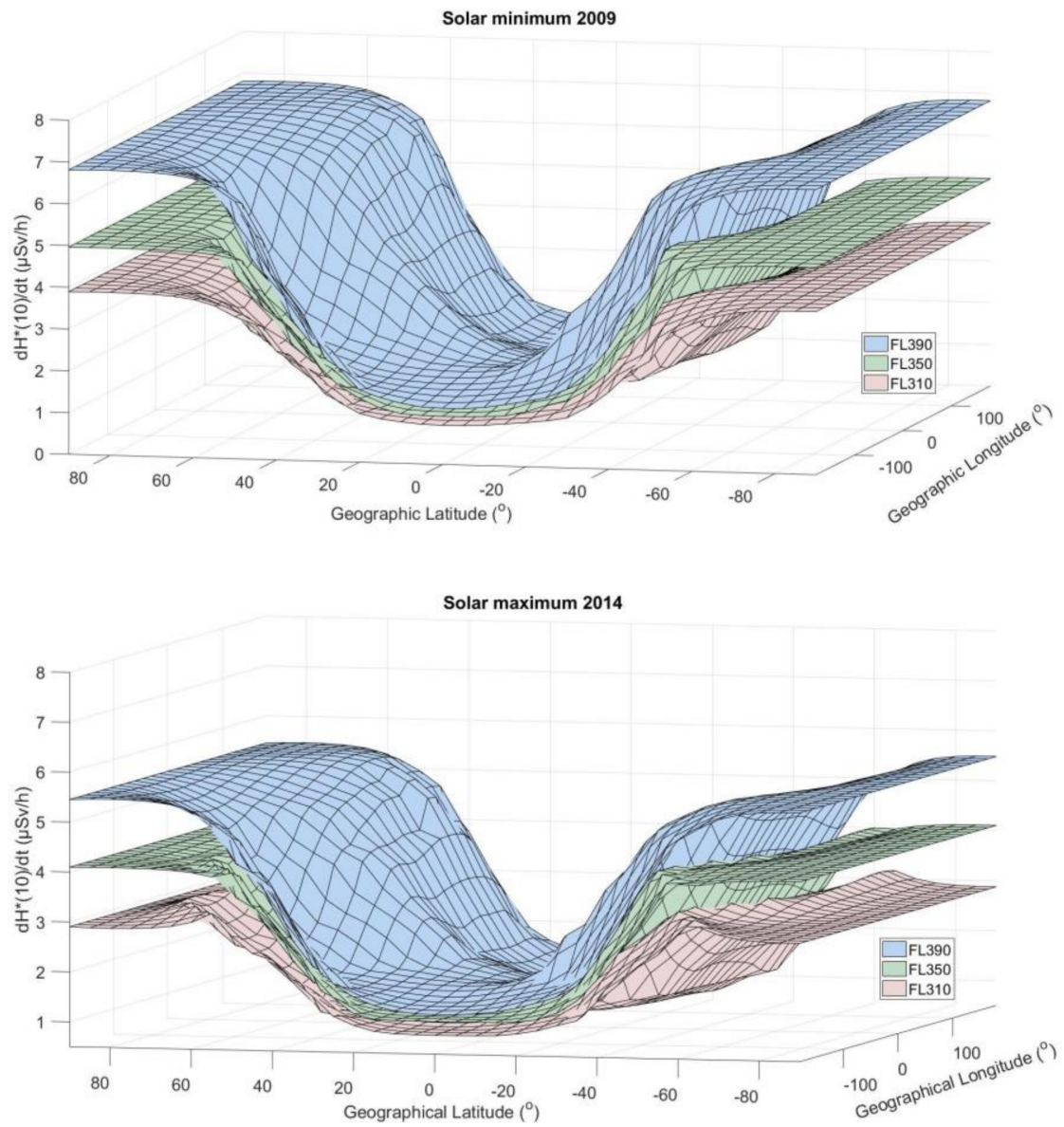

Figure 5. Ambient dose equivalent rate for different geographic coordinates for the years 2009 (solar minimum) and 2014 (solar maximum).
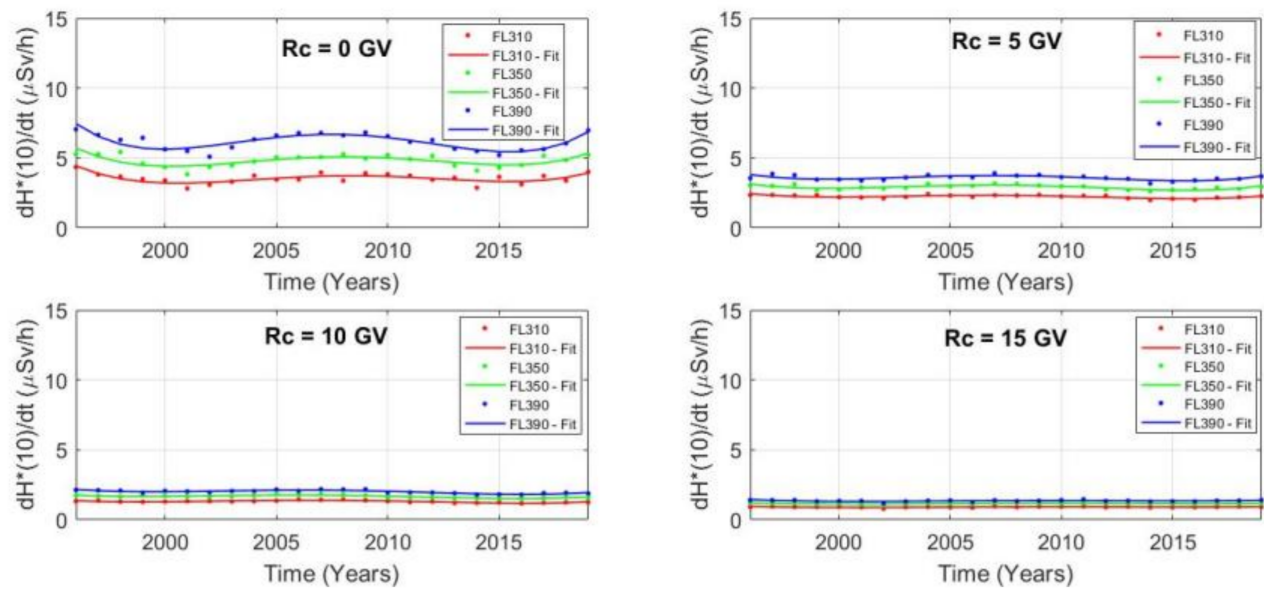

Figure 6. Ambient dose equivalent rate for the time period 1996-2019.

\section{Discussion and Conclusions}

In this work, a long-term analysis of the radiation exposure of aircrews during solar cycles 23 and 24 (1996-2019) has been performed. The novelty of this work is the extraction of results concerning the whole solar cycle 24 . The estimation of the operational quantity of ambient dose equivalent rate is based on a great number of Monte Carlo simulations, more specifically a number of 432 ones performed with the software tool DYASTIMA/DYASTIMA-R. Each simulation has been executed for a number of 
50000 events and 20 iterations. More specifically, dose rate variations are investigated as a function of the magnetic rigidity threshold, the phase of solar activity, and the altitude in the atmosphere.

According to the above analysis, it is observed that the polar regions $\left(\mathrm{Rc}_{\mathrm{c}}=0 \mathrm{GV}\right)$ are characterized by the greatest energy deposit and dose rate, while the middle geographic latitudes and the equatorial regions $(\mathrm{Rc}=15-17 \mathrm{GV})$ are characterized by lower values, which is due to the structure of the magnetosphere and the shielding of the geomagnetic field. The dose rate distribution is exponential in the whole range cut-off rigidity thresholds, a behavior that occurs in all phases of the solar cycle. The radiation dose in the different atmospheric layers is also anti-correlated with the solar activity, a fact that is more evident in the polar regions. This behavior decreases when approaching geographic latitude values close to the equator, where the dose rate becomes independent of the phase of the solar cycle. The dependence of the dose rate on solar activity in the polar regions is more pronounced at higher altitudes.

In the light of the up-to-date research concerning radiation exposure to cosmic rays, several well-known tools [6-9] have already been released. DYASTIMA/DYASTIMA-R provides an alternative procedure for the calculation of radiation exposure by considering the secondary cascades properties and accomplishes the aforementioned tools by confirming their results. In this sense, the user is able to study the air-shower characteristics as well as the radiation dose rate in the selected atmospheric layers for different scenarios. It is highlighted that the graphical user interface of DYASTIMA allows for the extensive parameterization of the characteristics of the planet and its atmosphere, and therefore it can be used for performing simulations in other planets, too. So far, it has been used for the study of the cascade's characteristics and the ionization rate in the atmosphere of Venus [26]. DYASTIMA has also been used for the determination of the cascades' characteristics during an extreme event, i.e., a ground level enhancement event [34].

In conclusion, DYASTIMA/DYASTIMA-R is a validated software tool according to international standards that provides all the critical parameters of the secondary particles' cascades description as well as accurate estimations for the radiation assessment of the exposure of pilots, flight attendants and frequent flyers due to cosmic radiation. This work summarizes the radiation dose rate due to the galactic component of cosmic radiation, which constitutes a permanent background of radiation exposure and is related to long-term effects on human health, as far as occupational exposure to cosmic radiation is concerned. More specifically, the simulations performed in this work provide calculations for specific points inside the Earth's atmosphere, which correspond to specific values of cut-off rigidity threshold, geographic coordinates, atmospheric altitude, and phase of solar cycle. A suitable mathematical manipulation of these results may allow for the reconstruction of a whole flight profile.

The next steps of this work include the study of high-intensity solar events, such as ground level enhancements (GLEs), which are solar energetic particles with energies greater than $433 \mathrm{MeV}$ that can be recorded by the ground-based neutron monitor network. These events are recorded at higher geographic latitudes, and they may be particularly harmful, since they may increase the radiation dose that aircrews and passengers are exposed to, especially during trans-polar flights in higher altitudes [35]. In order to prepare for extreme cosmic radiation events, it is necessary to have accurate and timely forecasting systems of such events (such as the GLE Alert system [36,37] and the HESPERIA UMASEP 500 [38]). A Space Weather Index D is also proposed (similar to the indexes for solar radiation storms and geomagnetic storms), regarding the levels of radiation exposure for aviators [39]. Last but not least, a combination of these results may be applied to various flight scenarios for the estimation of both the total dose and dose rate during a whole flight, by taking into account the flight path of the airplane and the total flight time.

Author Contributions: Conceptualization, M.D.; data curation, A.T. and A.S.; formal analysis, A.T.; investigation, A.T.; methodology, M.G. and D.A.; resources, A.K.; software, P.P.; supervision, H.M.; 
validation, P.K.; writing - review and editing, N.C. All authors have read and agreed to the published version of the manuscript.

Funding: This research received no external funding.

Institutional Review Board Statement: Not applicable.

Informed Consent Statement: Not applicable.

Data Availability Statement: The datasets generated and/or analysed during the current study are available from the corresponding author on reasonable request.

Acknowledgments: This work is supported by ESA SSA SWE Space Radiation Expert Service Centre activities (ESA contract number 4000113187/15/D/MRP). The European Neutron Monitor Services research is funded by the ESA SSA SN IV-3 Tender: RFQ/3-13556/12/D/MRP. A.Ne.Mo.S is supported by the Special Research Account of Athens University (70/4/5803). A.T. thanks the Hellenic State Scholarship Foundation (IKY) for supporting her with a doctoral scholarship through the Operational Programme "Human Resources Development, Education and Lifelong Learning" in the context of the project "Strengthening Human Resources Research Potential via Doctorate Research-2nd Cycle" (MIS-5000432), co-financed by Greece and the European Union (European Social Fund-ESF).

Conflicts of Interest: The authors declare no conflict of interest.

\section{References}

1. Beck, P.; Latocha, M.; Dorman, L.; Pelliccioni, M.; Rollet, S. Measurements and simulations of the radiation exposure to aircraft crew workplaces due to cosmic radiation in the atmosphere. Radiat. Prot. Dosim. 2007, 126, 564-567. [CrossRef] [PubMed]

2. Meier, M.M.; Copeland, K.; Kloble, K.E.J.; Matthia, D.; Plettenberg, M.C.; Schennetten, K.; Wirtz, M.; Hellweg, C.E. Radiation in the atmosphere. A hazard to aviation safety? Atmosphere 2020, 11, 1358. [CrossRef]

3. National Council on Radiation Protection and Measurements. Ionizing Radiation Exposure of the Population of the United States; NCRP Technical Report No. 160; NCRP: Bethesda, MD, USA, 2009.

4. European Commission. Directive 96/29/EURATOM of 13 May 1996 Laying Down Basic Safety Standards for the Protection of the Health of Workers and the General Public Against the Dangers Arising from Ionizing Radiation; Publications Office: Luxembourg, 1996.

5. European Commission. Comparison of Codes Assessing Radiation Exposure of Aircraft Crew Due to Galactic Cosmic Radiation, DirectorateGeneral for Energy, Directorate D—Nuclear Safety \& Fuel Cycle; Unit D4—Radiation Protection; Publications Office: Luxembourg, 2012.

6. Latocha, M.; Beck, P.; Rollet, S. AVIDOS-A software package for European accredited aviation dosimetry. Radiat. Prot. Dosim. 2009, 136, 286-290. [CrossRef] [PubMed]

7. SIEVERT. Available online: https://www.sievert-system.org/ (accessed on 20 October 2021).

8. Copeland, K. CARI-7A: Development and validation. Radiat. Prot. Dos. 2017, 175, 419-431. [CrossRef] [PubMed]

9. Mertens, C.J.; Meier, M.M.; Brown, S.; Norman, R.B.; Xu, X. NAIRAS aircraft radiation model development, dose climatology, and initial validation. Space Weather 2013, 11, 603-635. [CrossRef]

10. SPENVIS. Available online: https://www.spenvis.oma.be/ (accessed on 20 October 2021).

11. Paschalis, P.; Mavromichalaki, H.; Dorman, L.I.; Plainaki, C.; Tsirigkas, D. Geant4 software application for the simulation of cosmic ray showers in the Earth's atmosphere. New Astron. 2014, 33, 26-37. [CrossRef]

12. Agostinelli, S.; Allison, J.; Amako, K.A.; Apostolakis, J.; Araujo, H.; Arce, P.; Asai, M.; Axen, D.; Banerjee, S.; Barrand, G.; et al. Geant4-A simulation toolkit. Nucl. Instrum. Methods A 2003, 506, 250-303. [CrossRef]

13. Allison, J.; Amako, K.; Apostolakis, J.; Araujo, H.; Dubois, P.A.; Asai, M.; Barrand, G.; Capra, R.; Chauvie, S.; Chytracek, R.; et al. Geant4 developments and applications. IEEE Trans. Nucl. Sci. 2006, 53, 270-278. [CrossRef]

14. Allison, J.; Amako, K.; Apostolakis, J.; Arce, P.; Asai, M.; Aso, T.; Bagli, E.; Bagulya, A.; Banerjee, S.; Barrand, G.; et al. Recent developments in Geant4. Nucl. Instrum. Methods A 2016, 835, 186-225. [CrossRef]

15. Paschalis, P.; Tezari, A.; Gerontidou, M.; Mavromichalaki, H.; Nikolopoulou, P. Space Radiation Exposure Calculations during Different Solar and Galactic Cosmic Ray Activities. arXiv 2016, arXiv:1612.08937.

16. Paschalis, P.; Tezari, A.; Gerontidou, M.; Mavromichalaki, H.; Karaiskos, P. Radiation exposure of aircrews due to Space Radiation. HNPS Adv. Nucl. Phys. 2019, 26, 210-213.

17. International Commission on Radiological Protection. Radiological protection from cosmic radiation in aviation. Ann. ICRP 2016, $45,132$.

18. International Commission on Radiation Units and Measurements. Reference data for the validation of doses from cosmic-radiation exposure of aircraft crew. ICRU Report 84. J. Int. Comm. Radiat. Units Meas. 2010, 10.

19. ESA. ESA SSA P3 SWE-III Acceptance Test Report, R.137 Dynamic Atmospheric Tracking Interactive Model Application (DYASTIMA); ESA: Paris, France, 2019.

20. Tezari, A.; Paschalis, P.; Mavromichalaki, H.; Karaiskos, P.; Crosby, N.; Dierckxsens, M. DYASTIMA: Simulating air showers in the atmosphere of a planet. In Proceedings of the 70th IAC, Washington, DC, USA, 21-25 October 2019. 
21. Tezari, A.; Paschalis, P.; Mavromichalaki, H.; Karaiskos, P.; Crosby, N.; Dierckxsens, M. Assessing Radiation Exposure Inside The Earth's Atmosphere. Radiat. Prot. Dos. 2020, 190, 427-436. [CrossRef]

22. Athens Cosmic Ray Group. DYASTIMA Software User Manual. 2019. Available online: http://cosray.phys.uoa.gr/apps/ DYASTIMA/DYASTIMA_USER_MANUAL.pdf (accessed on 17 January 2022).

23. Paouris, E.; Mavromichalaki, H.; Belov, A.; Guischina, R.; Yanke, V. Galactic cosmic ray modulation and the last solar minimum. Sol. Phys. 2012, 280, 255-271. [CrossRef]

24. ISO 2533:1975; ISO Standard Atmosphere. International Organization for Standardization: Geneva, Switzerland, 2007.

25. Sanz Fernandez de Cordoba, S. 100 km altitude boundary for astronautics. Federation Aeronautique Internationale: Astronautic Records Commission (ICARE). 2004. Available online: https://www.fai.org/page/icare-boundary (accessed on 1 March 2021).

26. ISO 15390:2004; ISO Space Environment (Natural and Artificial)—Galactic Cosmic Ray Model. International Organization for Standardization: Geneva, Switzerland, 2004.

27. Plainaki, C.; Paschalis, P.; Grassi, D.; Mavromichalaki, H.; Andriopoulou, M. Interactions of cosmic rays with the Venusian atmosphere during different solar activity conditions. Ann. Geophys. 2016, 34, 595-608. [CrossRef]

28. Smart, D.F.; Shea, M.A. World grid of calculated cosmic ray vertical cutoff rigidities for epoch 2000.0. In Proceedings of the 30th International Cosmic Ray Conference, Yucatán, Mexico, 3-11 July 2007.

29. Gerontidou, M.; Katzourakis, N.; Mavromichalaki, H.; Yanke, V.; Eroshenko, E. World grid of cosmic ray vertical cut-off rigidity for the last decade. Adv. Space Res. 2021, 67, 2231-2240. [CrossRef]

30. Forbush, S. Cosmic ray intensity variations during two solar cycles. J. Geophys. Res. 1958, 63, 651-669. [CrossRef]

31. Singh, A.K.; Singh, D.; Singh, R.P. Impact of galactic cosmic rays on earth's atmosphere and human health. Atmos. Environ. 2011, 45, 3806-3818. [CrossRef]

32. Meier, M.M.; Hubiak, M.; Matthia, D.; Wirtz, M.; Reitz, G. Dosimetry at aviation altitudes (2006-2008). Radiat. Prot. Dos. 2009, 136, 251-255. [CrossRef] [PubMed]

33. Mrigakshi, A.I.; Matthiä, D.; Berger, T.; Reitz, G.; Wimmer-Schweingruber, R.F. Estimation of galactic cosmic ray exposure inside and outside the Earth's magnetosphere during the recent solar minimum between solar cycles 23 and 24. Adv. Space Res. 2013, 52, 979-998. [CrossRef]

34. Dorman, L.I.; Paschalis, P.; Plainaki, C.; Mavromichalaki, H. Estimation of the cosmic ray ionization in the Earth's atmosphere during GLE71. In Proceedings of the 34th International Cosmic Ray Conference, Hague, The Netherlands, 30 July-6 August 2015.

35. Mishev, A.L.; Usoskin, I.G. Assessment of the radiation environment at commercial jet-flight altitudes during GLE 72 on 10 September 2017 using neutron monitor data. Space Weather 2018, 16, 1921-1929. [CrossRef]

36. Souvatzoglou, G.; Papaioannou, A.; Mavromichalaki, H.; Dimitroulakos, J.; Sarlanis, C. Optimizing the real-time ground level enhancement alert system based on neutron monitor measurements: Introducing GLE Alert Plus. Space Weather 2014, 12, 633-649. [CrossRef]

37. Mavromichalaki, H.; Gerontidou, M.; Paschalis, P.; Paouris, E.; Tezari, A.; Sgouropoulos, C.; Crosby, N.; Dierckxsens, M. RealTime Detection of the Ground Level Enhancement on 10 September 2017 by A.Ne.Mo.S.: System Report. Space Weather 2018, 16, 1797-1805. [CrossRef]

38. Núñez, M.; Reyes-Santiago, P.J.; Malandraki, O.E. Real-time prediction of the occurrence of GLE events. Space Weather 2017, 15, 861-873. [CrossRef]

39. Meier, M.M.; Matthiä, D. A space weather index for the radiation field at aviation altitudes. J. Space Weather Space Clim. 2014, 4, A13. [CrossRef] 